\title{
The Solar Tachocline: Limiting Magneto-Tipping Instabilities
}

\author{
P. S. Cally \\ Centre for Stellar and Planetary Astrophysics, School of Mathematical \\ Sciences, Monash University, Victoria, Australia 3800
}

M. Dikpati

High Altitude Observatory, NCAR, Boulder, 3450 Mitchell Lane, CO 80301, USA

\section{P. A. Gilman}

High Altitude Observatory, NCAR, Boulder, 3450 Mitchell Lane, CO 80301, USA

\begin{abstract}
Two dimensional magneto-shear instabilities in the solar tachocline have been extensively explored in recent years. One of their most notable traits over a wide range of shear and magnetic profiles is a propensity for the magnetic field to tip substantially from its initial axisymmetric configuration, with possible implications for patterns of flux emergence. However, it is found that modifications of the standard models to include either kinetic and magnetic drag, or prograde toroidal velocity jets associated with magnetic bands, can suppress the instabilities, or considerably reduce their nonlinear development. In the case of tip reduction by jets, for a toroidal field of around $100 \mathrm{kG}$ in the tachocline (required for sunspots to emerge in sunspot latitudes), simulations indicate that jets capable of reducing tipping below the limits of detection from sunspot patterns at the surface are potentially detectable by helioseismic methods, and should be looked for. Establishing an upper limit to the jet may result in a lower limit for the amount of tipping to be expected.
\end{abstract}

\section{Introduction}

Helioseismic inversions imply that there exists a sharp shear layer at the base of the solar convection zone, of thickness $<0.05 R_{\odot}$, where the differential rotation of the convection zone transforms to a solid body rotation characteristic of the core (Brown et al. 1989; Kosovichev 1996; Charbonneau et al. 1997). This thin layer, called the tachocline, contains strong radial shear. The strongest toroidal fields of the Sun are believed to be generated in this layer through the $\Omega$-effect of a dynamo process (Dikpati et al. 2002). These fields are supposed to produce bipolar spots by rising to the surface due to their magnetic buoyancy (Choudhuri \& Gilman 1987; Fan, Fisher \& De Luca 1993; Caligari et al. 1995). 
The latitudinal profiles of these dynamo-generated toroidal fields in the tachocline are not known, but there are enough reasons (Harvey 1977; Zwaan 1992) to believe that toroidal fields should exist there in banded forms, at least at certain phases of the solar cycle. Noting the observational fact (Howard, Gilman \& Gilman 1984; Bogdan et al. 1988) that active regions have latitudinal widths typically in the range $6^{\circ}-10^{\circ}$, we (Cally, Dikpati \& Gilman 2003) have studied the nonlinear evolution of $2 \mathrm{D}$ magneto-tipping instabilities of $10^{\circ}$ toroidal bands placed at various latitudes in the solar tachocline. Using a solar-like tachocline latitudinal differential rotation $\left(\omega_{0}=r-s \mu^{2}\right.$, where $r$ is the equatorial rate, $s$ measures the pole-to-equator differential rotation, $\mu$ represents sine-latitude), we have found the following:

1. The dominant mode of instability in the tachocline is for longitude wavenumber $m=1$, which represents tipping of the band;

2. The strong toroidal band of several tens of $\mathrm{kG}$ peak field tips;

3. The higher its latitude location the more it tips;

4. $m>1$ modes occur for much weaker $(<25 \mathrm{kG})$ toroidal bands and cause 'deformation' of the band.

Recent theoretical models and simulations (Rempel et al. 2000; Dikpati \& Gilman 2001; Rempel \& Dikpati 2003) suggest that a toroidal band can achieve equilibrium against its poleward slip either by having extra mass on its poleward side or by having a prograde jet inside the band. Motivated by these studies, we investigate the effect of a jet in limiting the tipping of the toroidal band, using the 2D model of Cally, Dikpati \& Gilman (2003).

\section{Model}

In view of the strongly stable stratification in the radiative tachocline, our models are restricted to a spherical surface. We place Gaussian bands of toroidal magnetic field with $10^{\circ}$ band-width at specified latitudes, and postulate a prograde jet with angular velocity

$$
\omega_{j}=\sqrt{\left(\omega_{c}+\omega_{s}\right)^{2}+\alpha_{0}^{2}}-\left(\omega_{c}+\omega_{s}\right)
$$

where $\omega_{c}$ is the core rotation rate, $\omega_{s}$ represents a solar-like differential rotation with respect to the core, and $\alpha_{0} \sqrt{1-\mu^{2}}$ corresponds to the toroidal magnetic field profile in latitude. Here $\mu$ is the sine-latitude, and $\alpha_{0}(\mu)$ is the angular velocity of an Alfvén wave travelling on the toroidal band.

\subsection{Linear Instabilities}

The two frames in Figure 1 reveal the stabilizing effect of a prograde jet. The jet has a profound stabilizing influence on the magneto-tipping instability: the additional concentration of angular momentum inside the toroidal band gyroscopically inhibits 'tipping'. We note that, for given field strength parameter $a$ (where $a=1$ corresponts to a peak field of $10^{5} \mathrm{G}$ ), a jet can totally stabilize the 

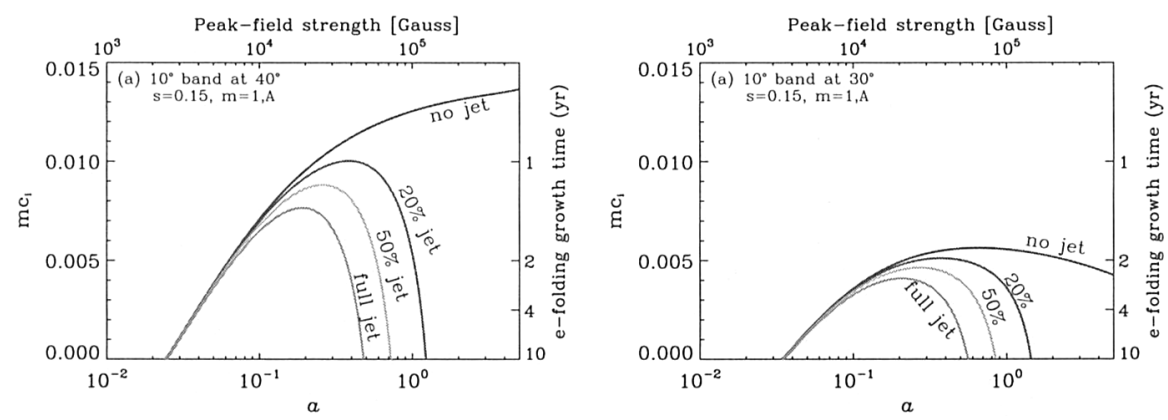

Figure 1. Growth rate curves $m c_{i}$ for a toroidal band, placed at two selected latitudes $\left(40^{\circ}\right.$ and $\left.30^{\circ}\right)$ in the tachocline, with full, partial and no jets. Temporal and longitudinal dependence is assumed to be of the form exp $i m(\theta-c t)$, where $c=c_{r}+i c_{i}$ is the (complex) phase speed. In this case $m=1$, corresponding to the tipping modes.

system against tip. But even if the jet is not strong enough to achieve stability, it may substantially reduce the growth rate. It is also found that, the higher the latitude location of the band, the more the influence of the jet.

\subsection{Nonlinear Evolution}

The nonlinear evolution of unstable 2D magneto-shells is followed using the spectral code of Cally (2001). Figure 2 (left panel) reveals that the tachocline latitudinal differential rotation naturally evolves a jet (despite having none initially) of about $20 \%$ amplitude with respect to the total pole-to-equator differential rotation amplitude as a result of nonlinear evolution of the tipping instability.
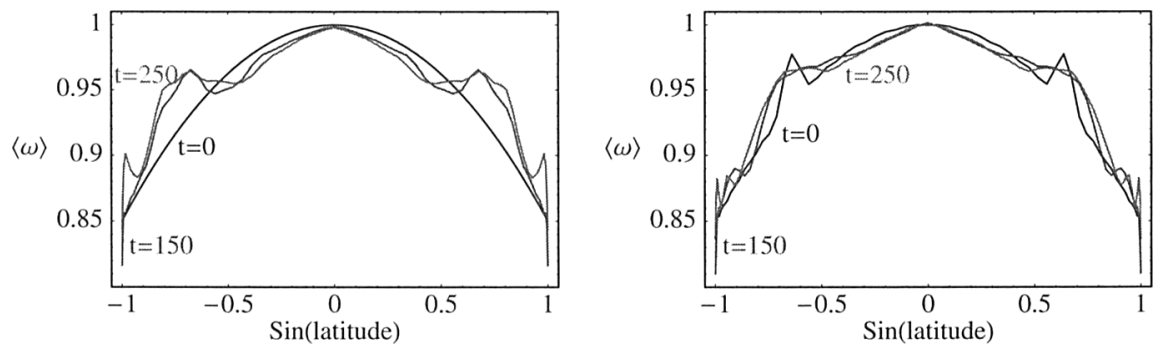

Figure 2. Evolution of the longitudinally averaged tachocline differential rotation $\langle\omega\rangle$ as a result of the magneto-tipping instability of a toroidal band (placed at $40^{\circ}$ latitude) without a jet (left) and with a $20 \%$ jet (right). $t=100$ in dimensionless units corresponds to about 1 year.

From Figure 2 (right panel) we also find that an initially imposed tachocline latitudinal differential rotation jet of about $20 \%$ maintains that state, reconfirming that it is one of the steady states for tachocline latitudinal differential rotation. The presence of the jet limits the tipping (Figures 3 and 4 ). The 
higher the latitude location of the band, the more is the influence of the jet in inhibiting tipping.
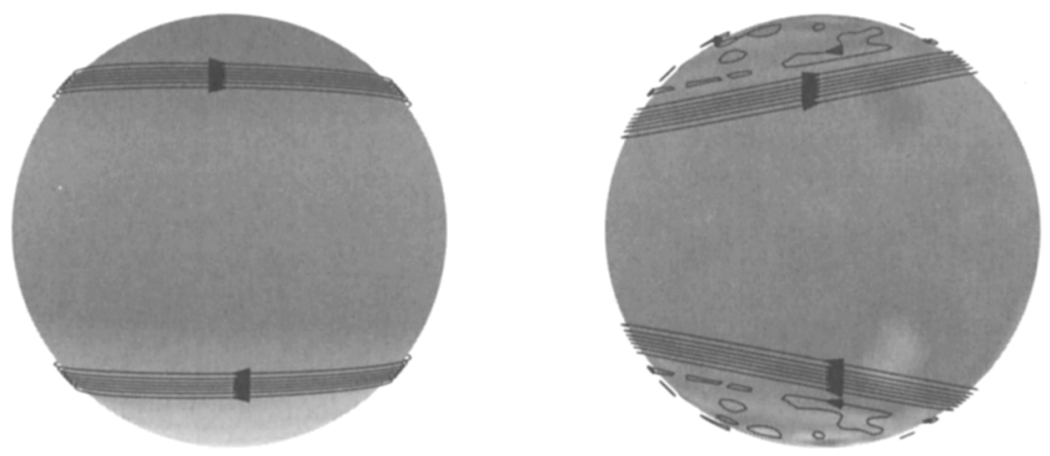

Figure 3. Left frame shows the initial configuration of a toroidal magnetic band (with slight $m=1$ perturbation) in the $2 \mathrm{D}$ tachocline. Right frame shows how the band tips as a result of undergoing this instability for a few years (without an initial jet).
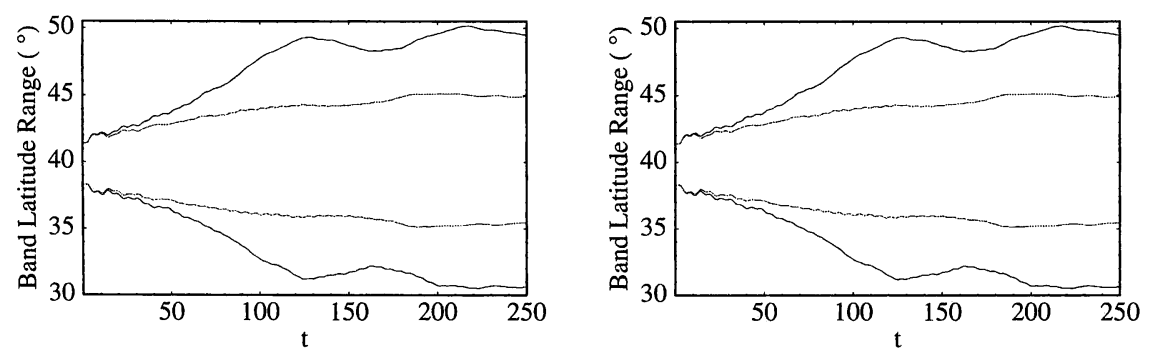

Figure 4. Calculated 'tip' angle of a toroidal band without a prograde jet (dark curves) and with a $20 \%$ jet (light curves). Left frame represents the case for a toroidal band at $40^{\circ}$ and the right frame that for a band at $30^{\circ}$.

\section{Discussion}

We have shown that velocity jets in toroidal magnetic bands in the radiative solar tachocline act to stabilize the bands against tipping instabilities explored by Cally, Dikpati and Gilman (2003). Without such stabilizing influences, field tip of order $20^{\circ}$ is predicted, and yet there is (as yet) no clear observational evidence for this. Rather indirectly, this argues in favour of the presence of tachocline jets. However, clearly, more direct confirmation is required. 
In large part, the purpose of this paper is to excite interest in the helioseismic community in searching for jets below the base of the convection zone. This is of interest both because of the stability implications, and also because they may be a proxy for the magnetic bands themselves, which are probably more difficult to detect directly. Questions associated with helioseismic detection of jets include:

1. Can the jet be observed through helioseismology?

2. Can the method and kernels of Christensen-Dalsgaard et al. (1996) be improved to detect a jet in the solar tachocline, and in particular, how sharp (both in radius and latitude) do the kernels need to be?

3. How might non-axisymmetry (e.g., due to tipping) affect detection?

4. Are local helioseismic techniques better suited than global methods to finding jets?

Acknowledgments. This work has been supported by NASA grants W19752, W-10107 and W-10175. One of us (P. S. Cally) thanks High Altitude Observatory of NCAR for supporting his visit during this northern spring. The National Center for Atmospheric Research is sponsored by the National Science Foundation.

\section{References}

Bogdan, T. J., Gilman, P. A., Lerche, I., \& Howard, R. F. 1988, ApJ, 327, 451

Brown, T. M., Christensen-Dalsgaard, J., Dziembowski, W. A., Goode, P. R., Gough, D. O., \& Morrow, C. A. 1989, ApJ, 343, 526

Caligari, P., Moreno-Insertis, F., \& Schüssler, M. 1995, ApJ, 441, 886

Cally, P. S. 2001, Solar Phys., 199, 231

Cally, P. S., Dikpati, M., \& Gilman, P. A. 2003, ApJ, 582, 1190

Choudhuri, A. R., \& Gilman, P. A 1987, ApJ, 316, 788

Christensen-Dalsgaard, J., Howe, R., Schou, J., Thompson, M. J., Toomre, J. 1996, in Provost J. and Schmider F.-X. eds., Sounding Solar and Stellar Interiors, IAU Symposium, 181

Dikpati, M., Corbard, T., Thompson, M. J., \& Gilman, P. A 2002, ApJ, 575, L41

Dikpati, M., \& Gilman, P.A 2001, ApJ, 552, 348

Fan, Y., Fisher, G. H., \& DeLuca, E. E. 1993, ApJ, 405, 390

Harvey, J. 1977, in E. A. Müller (ed.), Highlights of Astronomy, 4, 223

Howard, R. F., Gilman, P. I., \& Gilman, P. A. 1984, ApJ, 283, 373

Rempel, M., \& Dikpati, M. 2003, ApJ, 584, 524

Rempel, M., Schüssler, M., \& Tóth, G. 2000, A\&A, 363, 789

Zwaan, C. 1992, in J. H. Thomas \& N. O. Weiss (eds.), Sunspots: Theory and Observations, 75 